\title{
Nécessité d'une stratégie globale avec des objectifs clairs
}

\section{Beatrix Meyer}

Cheffe de la division Médecine et tarifs hospitaliers

Avec SwissDRG version 7.0, SwissDRG SA a de nouveau entrepris de nombreux changements utiles. Il est cependant important de mettre sur pied une stratégie globale avec des objectifs clairs pour les versions futures afin d'éviter une éventuelle intervention du Conseil fédéral. Elle doit par ailleurs tenir compte de l'homogénéité médicale des DRG en plus de leur homogénéité économique.

\section{Eviter une intervention du Conseil fédéral}

Lors de son analyse des deux dernières versions de SwissDRG, le Conseil fédéral est à chaque fois parvenu à la conclusion que la structure tarifaire n'était pas assez différenciée. Il a par conséquent exigé une stratégie et un plan d'action "pour soit différencier la structure tarifaire jusqu'au point où un taux de base (base-

\section{Le nombre de cas coûteux et fortement} déficitaires est en baisse.

rate) uniforme serait possible, soit pouvoir utiliser de manière ordonnée des taux de base différenciés». ${ }^{1}$ Afin d'améliorer la structure tarifaire, SwissDRG SA a donc élaboré l'année passée un plan d'action qui prévoit une "valeur indicative pour une fourchette de calcul des coûts moyens par cas de $\pm 5 \% »{ }^{2}$ SwissDRG SA veut atteindre cet objectif d'ici à la fin 2019. Le Conseil fédéral a émis un avis critique à ce sujet, car la façon dont SwissDRG SA compte atteindre cet objectif est peu claire. A défaut d'un accord entre les partenaires tarifaires, le Conseil fédéral recommande d'utiliser la structure tarifaire SwissDRG de manière différenciée au moyen de la classification hospitalière de l'Office fédéral de la santé publique (OFSP). ${ }^{3}$ Du point de vue de la FMH, il s'agit d'éviter que ce modèle complexe de l'OFSP soit appliqué et d'éviter que les partenaires tarifaires perdent le contrôle de ce dossier. Quant à savoir dans quelle mesure SwissDRG SA s'est rapprochée de l'ob-

Vous trouverez I'analyse détaillée de SwissDRG version 7.0 dans la prise de position FMH: www.fmh.ch $\rightarrow$ Tarifs hospitaliers $\rightarrow$ Positions $\rightarrow$ Prises de position. jectif d'une "valeur indicative pour une fourchette de calcul des coûts moyens par cas de $\pm 5 \%$ » avec la version 7.0 de SwissDRG, la question reste ouverte.

\section{Nécessité d'une stratégie globale et d'une documentation transparente}

Avec SwissDRG version 7.0, on ne voit toujours pas clairement quelle est l'orientation générale suivie par SwissDRG SA pour le développement, ni comment les différentes transformations s'intègrent dans le contexte global. C'est pourquoi il est demandé à SwissDRG SA d'élaborer, avec la participation de ses partenaires, une stratégie globale avec des objectifs clairs. Celle-ci devra notamment montrer par quelles mesures SwissDRG SA entend atteindre l'objectif d'une "valeur indicative pour une fourchette de calcul des coûts moyens par cas de $\pm 5 \%$ \%. Une stratégie de développement claire et une documentation transparente des travaux effectués et des objectifs de développement atteints (ou non) seraient utiles pour l'utilisateur.

\section{De nombreux changements utiles et plus de rétributions additionnelles}

SwissDRG SA a de nouveau procédé à diverses transformations utiles. A cette occasion, 72 DRG ont été supprimés, et 75 DRG nouvellement admis. La version 7.0 de SwissDRG présente désormais 1041 DRG. Un point positif est que, pour les adaptations, SwissDRG SA ne s'est pas limitée au niveau des DRG particuliers, mais a tenu compte pour ce faire du contexte global de la $\mathrm{MDC}^{4}$. SwissDRG SA a entrepris des adaptations dans presque toutes les MDC. 
Cela dit, selon la FMH, il ne faudrait pas entreprendre des changements sur la base de variations statistiques minimes. Car, suivant l'état des données, les modifications qui en résultent doivent être recorrigées l'année d'après. On obtient alors, par exemple pour les coûts relatifs (cost-weight), de fréquentes variations qui pourraient être évitées. A ce propos, un nouveau relèvement des exigences envers la qualité des données serait utile. Le fait que SwissDRG SA se réfère aux données de plusieurs années pour le calcul de quelques DRG au nombre de cas réduit est positif. SwissDRG SA pourrait également tester ce procédé pour des DRG qui présentent des variations fréquentes de coûts relatifs. En outre, il est réjouissant que le nombre de rémunérations supplémentaires pour les médicaments, produits

\section{Veiller à l'homogénéité médicale et écono- mique d'un DRG.}

sanguins et procédures onéreuses ait augmenté à un total de 93. Ces rémunérations supplémentaires contribuent à une rétribution correcte, sans faire augmenter les coûts globaux.

\section{Veiller à la transparence médicale}

Lors de transformations de DRG, il faut tenir compte des aspects économiques, mais aussi des critères médicaux. Certes, SwissDRG SA prend parfois en considération les aspects médicaux. Ainsi, par exemple dans les modifications de la MDC 05 Maladie et troubles de l'appareil circulatoire, les interventions médicales comparables ont été réunies de façon judicieuse et avec clarté. ${ }^{5}$ Mais il y a aussi des exemples où la logique médicale n'a pas été prise en considération. Dans ces cas, les coûts et les durées de séjour comparables ont été les seules raisons d'une transformation de DRG. ${ }^{6}$ Pour les versions futures de SwissDRG, la FMH recommande de veiller à l'homogénéité non seulement économique, mais aussi médicale.

\section{Continuer d'améliorer la prise en compte des cas fortement déficitaires}

L'année passée, le Conseil d'administration de SwissDRG SA a décidé que la prise en compte des traitements coûteux et fortement déficitaires dans le système SwissDRG se ferait en poursuivant le développement et la différenciation de celui-ci. Pour SwissDRG version 7.0, SwissDRG SA a mis l'accent sur la prise en compte des cas de soins intensifs et de soins intermédiaires, des interventions en plusieurs temps ainsi que des traitements complexes. Elle a utilisé, pour son évaluation, les cas dont le déficit est $>40000 \mathrm{CHF}$ et, à juste titre, elle n'a pas tenu compte du volume total des coûts de chaque cas concerné. Tant le nombre de cas fortement déficitaires de l'ensemble des hôpitaux que le volume de déficit qui en résulte ont diminué. Ce volume total de déficit des hôpitaux a baissé d'environ un quart entre la version 5.0 et la version 7.0 de SwissDRG. Si l'on considère séparément les cas fortement déficitaires des hôpitaux universitaires, le volume de déficit a également baissé d'un quart au cours de la même période, bien qu'il s'élève encore à près de 100 millions de francs. Pour les autres catégories d'hôpitaux, le problème n'est toujours pas réglé. C'est pourquoi il faut poursuivre les efforts en vue d'une prise en charge appropriée des cas fortement déficitaires.

\section{Conclusions et recommandations pour les versions futures}

Avec ses nombreuses transformations, SwissDRG SA a poursuivi l'amélioration de la structure tarifaire. Les adaptations ont souvent été motivées par les coûts et les durées de séjour. Les aspects médicaux sont malheureusement passés en arrière-plan. Il faudra de nouveau plus en tenir compte dans les futures versions. Quant à savoir quel concept global SwissDRG SA a suivi dans ses transformations, la question est sans réponse. Il est clair que, l'année passée, SwissDRG SA a élaboré un plan d'action succinct sous la pression du Conseil fédéral. Dans ce plan, elle a fixé l'objectif d'une «valeur indicative pour une fourchette de calcul des coûts

\section{Elaborer une stratégie globale et éviter une} intervention du Conseil fédéral.

moyens par cas de $\pm 5 \%$ ». Par contre, on ne sait pas par quelles mesures SwissDRG SA entend atteindre cet objectif pour la fin 2019. Par conséquent, la FMH recommande à SwissDRG SA d'élaborer d'entente avec ses partenaires une stratégie globale avec des objectifs clairs, en incluant aussi cet objectif et les mesures prévues. En outre, SwissDRG SA devrait régulièrement montrer où en est la progression par rapport aux objectifs. Il y a un besoin d'agir évident pour éviter une éventuelle intervention du Conseil fédéral aussi dans le secteur hospitalier. 